\title{
PRODUÇÃO CIENTÍFICA BRASILEIRA ACERCA DA SAÚDE DO TRABALHADOR NAS TESES E DISSERTAÇÕES DE ENFERMAGEM
}

\section{BRAZILIAN SCIENTIFIC PRODUCTION ON THE HEALTH OF THE WORKER IN THE THESES AND NURSING DISSERTATIONS}

\section{LA PRODUCCIÓN CIENTÍFICA BRASILEÑA ACERCA DE LOS TRABAJADORES DE LA SALUD EN TESIS Y DISERTACIONES DE ENFERMERÍA}

\author{
Sabrina de Souza Gurgel ${ }^{1}$, Érica Oliveira Matias ${ }^{2}$, Maria Iracema Capistrano Bezerra ${ }^{3}$, \\ Francisca Elisângela Teixeira Lima ${ }^{4}$, Ires Lopes Custódio ${ }^{5}$
}

\begin{abstract}
RESUMO
Objetivo: descrever o panorama da produção científica desenvolvida acerca da saúde do trabalhador pela Enfermagem brasileira nos cursos de mestrado e doutorado, os quais foram informados e catalogados entre os anos de 2009 a 2014. Método: estudo descritivo, documental, quantitativo, desenvolvido a partir seis catálogos do Centro de Estudos e Pesquisas em Enfermagem, compreendendo os resumos das dissertações e teses de enfermagem desenvolvidas no período de 2009 a 2014 nas universidades brasileiras. Resultados: detectou-se 161 estudos desenvolvidos acerca da saúde do trabalhador, predominando: dissertações $(73,91 \%)$, realizados na Região Sudeste $(62,8 \%)$, em hospitais (70,8\%); cujos sujeitos do estudo foram a equipe de enfermagem $(52,2 \%)$. Conclusão: há estudos contínuos sobre saúde do trabalhador ainda que se evidenciem disparidades regionais no processo de produção desse conhecimento.

Descritores: Saúde do trabalhador; Educação de pós-graduação em enfermagem; Indicadores de produção científica.
\end{abstract}

\begin{abstract}
Objective: To describe the panorama of the Brazilian scientific production developed on occupational health by the Nursing masters and doctorate courses informed and cataloged from 2009 to 2014. Method: It was a descriptive, documentary, quantitative study developed from six catalogs of the Center of Studies and Research in Nursing comprising the abstracts of nursing dissertations and theses developed from 2009 to 2014 in Brazilian universities. Results: 161 studies on occupational health were detected, predominantly dissertations (73.91\%), conducted in the Southeast Region (62.8\%), in hospitals (70.8\%), and whose

\footnotetext{
${ }^{1}$ Enfermeira. Mestranda em Enfermagem pela Universidade Federal do Ceará. Especialista em Enfermagem do Trabalho pela Universidade Estadual do Ceará. E-mail: sabrinagurgel@ hotmail.com

${ }^{2}$ Enfermeira do Instituto Federal do Ceará. Doutoranda em Enfermagem pela Universidade Federal do Ceará. Email: erica_enfermagem@yahoo.com.br

${ }^{3}$ Fisioterapeuta. Mestre em Saúde Coletiva pela Universidade de Fortaleza. Coordenadora Pedagógica da Residência Integrada em Saúde da Escola de Saúde Pública do Ceará. E-mail: iracemabezerra@ yahoo.com.br

4 Enfermeira. Doutora em Enfermagem pela Universidade Federal do Ceará. Professora Adjunto do Departamento de Enfermagem da UFC. E-mail: felisangela@yahoo.com.br

${ }^{5}$ Enfermeira do Hospital Dr. Carlos Alberto Studart Gomes. Doutoranda em Enfermagem pela Universidade Federal do Ceará. E-mail: iresl.custodio@gmail.com
} 
subjects were the nursing team $(52.2 \%)$. Conclusion: There are continuous studies on occupational health despite the evident regional disparities in this knowledge production process.

Descriptors: Occupational Health; Education, Nursing, Graduate; Scientific Publication Indicators.

\section{RESUMEN}

Objetivo: describir el panorama de la producción científica desarrollada en la salud del trabajador por la enfermería brasileña en máster y cursos de doctorado, que se informaron y catalogado entre los años 2009 a 2014. Métodos: estudio descriptivo, documental, cuantitativo, desarrollado a partir de seis catálogos del Centro de Estudios e Investigaciones en Enfermería, comprendiendo los resúmenes de las disertaciones y tesis de enfermería desarrolladas en el período de 2009 a 2014 en las universidades brasileñas. Resultados: Hemos detectado 161 estudios desarrollados sobre la salud ocupacional, predominando: disertaciones $(73,91 \%)$, llevadas a cabo en el Sudeste $(62,8 \%)$, hospitales $(70,8 \%)$; cuyo estudio los sujetos fueron el personal de enfermería (52,2\%). Conclusión: Hay estudios en curso sobre la salud del trabajador también que son evidentes las diferencias regionales en la producción de este proceso de conocimiento.

Descriptores: Salud laboral; Educación de posgrado en enfermería; Indicadores de producción científica.

\section{INTRODUÇÃO}

A partir da pesquisa científica são realizados estudos sobre os mais variados temas e feitas descobertas que contribuem para o desenvolvimento científico e tecnológico das profissões. As universidades desempenham importante papel neste desenvolvimento formando recursos humanos e produzindo conhecimento por meio de seus pesquisadores, principalmente aqueles que fazem parte dos programas de pós-graduação stricto sensu. ${ }^{1}$ No contexto da enfermagem, as atividades de pesquisa foram cruciais para o desenvolvimento científico da profissão e entre seus inúmeros temas estudados tem-se a saúde do trabalhador.

A saúde do trabalhador está inserida na Constituição Federal de 1988 como atribuição do Sistema Único de Saúde (SUS) e surgiu como uma área da Saúde Coletiva em que se estudam as relações entre o trabalho, a saúde e o ambiente. ${ }^{2}$ Esta lei foi introduzida desde a promulgação da Lei 8.080/90, a qual dispõe sobre as condições para a promoção, proteção e recuperação da saúde do trabalhador, com a preocupação em incluírem-se estratégias e ações visando a qualidade de vida do trabalhador no ambiente de trabalho. ${ }^{3}$

Atualmente é um dos temas atuais mais relevantes e está inserido na agenda nacional de prioridades em pesquisa e saúde do Ministério da Saúde desde 2008. ${ }^{4}$ A qualidade de vida do trabalhador vem despertando o interesse de estudiosos e dirigentes das empresas, como suporte para 
o binômio: satisfação da pessoa no trabalho e produção.

Observa-se que há evolução na quantidade de produção científica brasileira sobre saúde do trabalhador, uma vez que está seguindo a tendência das demais áreas do conhecimento. Dessa forma, o progresso observado na quantidade de trabalhos sobre saúde do trabalhador na saúde coletiva mostra o potencial de crescimento da área. ${ }^{5}$ Além disso, devido à busca incessante pela realização de diferentes atividades, há um aumento das cargas laborais no homem, levando ao aparecimento de doenças físicas, psíquicas e emocionais, o que leva à busca de evidências científicas sobre as formas de adoecimento pelo trabalho, bem como as formas para o enfrentamento e prevenção ao adoecimento e acidentes de trabalho. ${ }^{6}$

Diante dessas considerações, é importante que os enfermeiros realizem estudos sobre saúde e meio ambiente como uma integração aos temas vida, saúde e trabalho. Dessa forma, questiona-se: Que regiões brasileiras publicaram pesquisas científicas acerca dessa temática? Que instituições de saúde estão sendo contempladas? Quem são os sujeitos estudados nessas instituições?

Diante do exposto, a análise da produção científica em Saúde do Trabalhador, desenvolvida por enfermeiros, a partir de suas teses e Rev Enferm Atenção Saúde [Online]. Jan/Jun 2017; 6(1):130-139 dissertações, desperta a reflexão sobre a prática da Enfermagem e a construção de conhecimento nesse setor, estruturada nos distintos Programas de Pós-Graduação em que enfermeiros realizam sua formação. Assim, justifica-se a importância do estudo bibliométrico de produtos científicos dessa natureza, pois, através dele, é possível compreender os direcionamentos do setor de Saúde do Trabalhador, suas fortalezas e fragilidades, suas interfaces e possibilidades de avanços.

O estudo teve como objetivo descrever o panorama da produção científica desenvolvida acerca da saúde do trabalhador pela Enfermagem brasileira nos cursos de mestrado e doutorado, os quais foram informados e catalogados entre os anos de 2009 a 2014.

Espera-se que a resolução dessas interrogações possa aperfeiçoar o conhecimento dos enfermeiros na temática, sobretudo favorecer a ascensão da qualidade de vida dos trabalhadores da saúde nos ambientes de trabalho.

\section{MÉTODO}

Trata-se de um estudo bibliométrico, descritivo e quantitativo, com análise retrospectiva documental, desenvolvido a partir dos seis catálogos do Centro de Estudos e Pesquisas em Enfermagem (CEPEn), compreendendo os resumos das dissertações e teses de enfermagem 
desenvolvidas no período de 2009 a 2014 nas universidades brasileiras.

A detecção dos resumos com as referidas temáticas ocorreu da seguinte forma: acesso ao site da ABEn (www.abennacional.org.br); em seguida o link dos canais do CEPEn, no catálogo de teses e dissertações; e volumes dos catálogos: (volume XXVIII, 2009; volume XXIX, 2010; volume XXX, 2011; volume XXXI, 2012; volume XXXII, 2013; volume XXXIII, 2014).

Foram encontradas 2.688 dissertações (mestrado) e 947 teses (doutorado), que representaram o universo do estudo. A população foi composta de 161 estudos, os quais atenderam e apresentaram temáticas relacionadas à enfermagem do trabalho; saúde dos trabalhadores que trabalhavam nas instituições de saúde; acidente de trabalho; qualidade de vida, exposição a agentes biológicos e doenças ocupacionais. Excluíram-se estudos nos quais os sujeitos da pesquisa não eram trabalhadores da saúde.

A coleta de dados ocorreu no mês de janeiro de 2016. Para a seleção das teses e dissertações acerca da saúde do trabalhador, buscou-se pelos Descritores em Ciências da Saúde (DECS), cadastrados na Biblioteca Virtual em Saúde, os quais foram: saúde do trabalhador, enfermagem do trabalho, Rev Enferm Atenção Saúde [Online]. Jan/Jun 2017; 6(1):130-139 absenteísmo, acidentes de trabalho, condições de trabalho, doenças profissionais, esgotamento profissional, qualidade de vida e riscos ocupacionais.

Os resumos foram submetidos à leitura exploratória, seletiva e analítica, procurando identificar e responder os quesitos previamente determinados em um instrumento de coleta de dados produzido pelas autoras, tendo sido constatada, previamente, a adequação do mesmo para o alcance dos objetivos propostos. De posse da análise da produção mencionada, os resultados foram apresentados em tabelas, permitindo melhor visualização e compreensão do fenômeno pesquisado.

Os aspectos éticos e legais foram respeitados, dispensando-se o termo de consentimento dos sujeitos e avaliação do comitê de ética em pesquisa, já que as dissertações e teses analisadas são documentos públicos.

\section{RESULTADOS}

A partir da leitura e análise dos resumos encontrou-se o montante de 3.635 estudos produzidos por enfermeiros no Brasil, os quais foram informados e catalogados entre os anos de 2009 a 2014. Desse total, 161 correspondem ao objeto de pesquisa "Saúde do Trabalhador", compondo o total de $4,43 \%$ da produção nacional de enfermeiros. Desses, 119 
$(73,91 \%)$ referem-se às dissertações e 42 $(26,09 \%)$, às teses.

O Gráfico 1 apresenta a distribuição científica acerca da Saúde do Trabalhador, segundo ano de defesa (2006 a 2015).

em números absolutos da produção

Gráfico 1: Caracterização dos resumos publicados nos catálogos do CEPEn de 2009 a 2014/ano de defesa

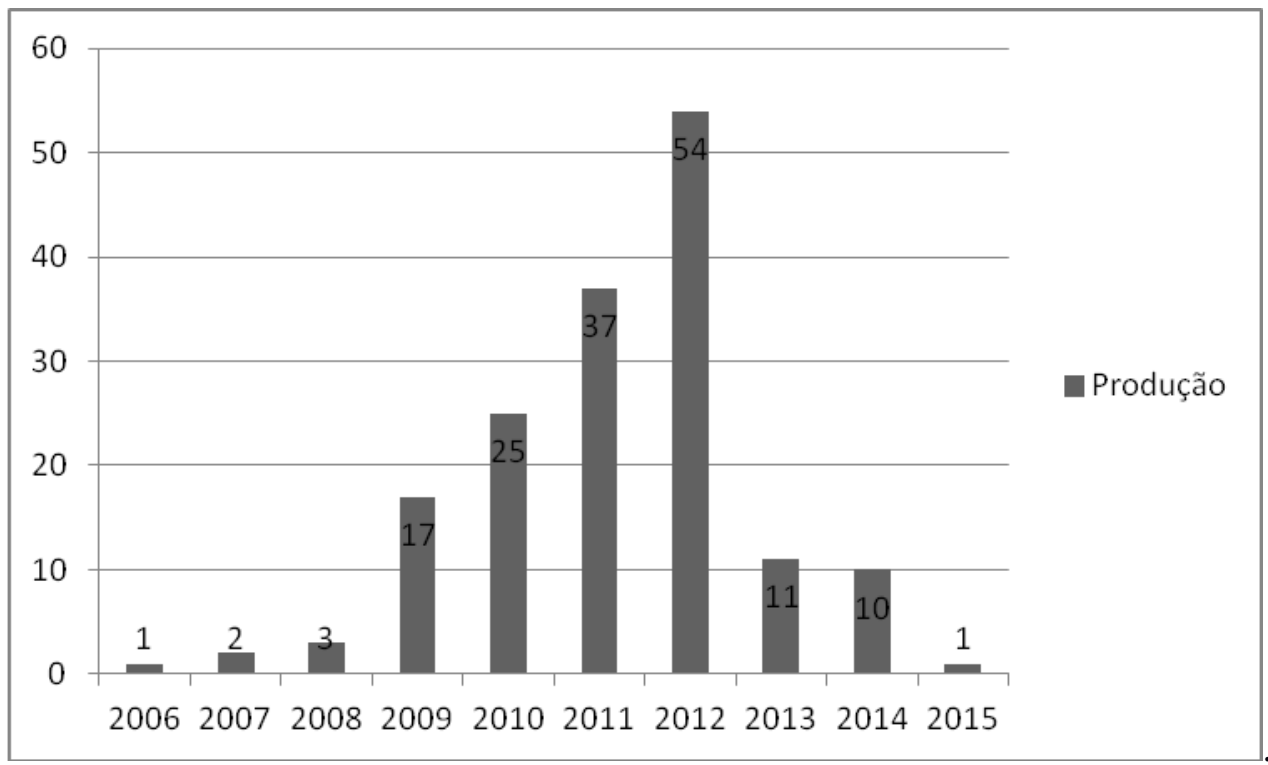

Os catálogos do CEPEn pesquisados são referentes ao período de 2009 a 2014, entretanto, cumpre destacar que o ano do catálogo refere-se ao ano em que os estudos foram recebidos, organizados e sistematizados pelo CEPEn, ou seja, não reflete necessariamente $o$ ano de publicação do estudo. Dessa forma, foram encontrados estudos de diversos anos de área geográfica.

\begin{tabular}{cccc}
\hline Região & Área geográfica & n & \% \\
\hline Sudeste & São Paulo & 55 & 34,2 \\
& Rio de Janeiro & 31 & 19,3 \\
& Minas Gerais & 15 & 9,3 \\
& & & \\
\multirow{2}{*}{ Sul } & Rio Grande do Sul & 13 & 8,1 \\
& Santa Catarina & 9 & 5,6 \\
& Paraná & 4 & 2,5
\end{tabular}


Nordeste

Bahia
Paraíba
Ceará
Pernambuco
Rio Grande do Norte
Piauí

$\begin{array}{ll}8 & 5,0 \\ 6 & 3,7 \\ 2 & 1,2 \\ 2 & 1,2 \\ 2 & 1,2 \\ 1 & 0,6\end{array}$

Centro-Oeste

Goiás

Distrito Federal

Mato Grosso

$\begin{array}{ll}7 & 4,4 \\ 4 & 2,5 \\ 1 & 0,6\end{array}$

$\frac{\text { Norte }}{\text { Fonte: Catálogos do CEPEn de 2009-2014. }}$

De acordo com a tabela 1 , no tocante às áreas geográficas do estudo, verificou-se que a maioria procede da Região Sudeste $(62,8 \%)$. A produção da Região Sul totalizou 26(16,2\%) trabalhos entre teses e dissertações. Os estudos apresentados em outras áreas incluem: na Região Nordeste 21(12,9\%) estudos, seguido da Região do
Centro-Oeste com $12(7,5 \%)$ e da Região Norte com apenas $1(0,6 \%)$ estudo.

$\mathrm{Na}$ tabela 2, estão expostos os resumos publicados nos catálogos do CEPEn de 2009-2014, referentes as instituições de saúde e os sujeitos da pesquisa.

Tabela 2 - Caracterização dos resumos publicados nos catálogos do CEPEn de 20092013/instituições de saúde e os sujeitos envolvidos

\begin{tabular}{lcc}
\hline \multicolumn{1}{c}{ Variáveis } & n & \% \\
\hline Instituição de saúde & 114 & 70,8 \\
Hospital & 19 & 11,8 \\
Unidade Básica de Saúde & 14 & 8,7 \\
Não especificado & 9 & 5,5 \\
SAMU & 2 & 1,3 \\
Unidade de Pronto-Atendimento & 2 & 1,3 \\
Clínica de Diálise & 1 & 0,6 \\
Centro de reabilitação psicossocial & & \\
& & \\
Sujeitos envolvidos & 84 & 52,2 \\
Equipe de enfermagem & 40 & 24,8 \\
Enfermeiros & 24 & 14,9 \\
Equipe de enfermagem e profissionais da saúde & 5 & 3,1 \\
Socorristas & 5 & 3,1 \\
Técnicos de enfermagem & 3 & 1,9 \\
Técnicos e auxiliares de enfermagem &
\end{tabular}

Fonte: Catálogos do CEPEn de 2009-2014. 
Entre os trabalhos publicados no período considerado, 14 autores não especificaram no resumo o local em que foi realizada a coleta de dados. Entre os que citaram, a maioria das coletas ocorreu em hospital, sendo 114(70,8\%), seguido por unidades básicas de saúde (11,8\%), pelo SAMU (5,5\%), unidades de prontoatendimento e clínicas de diálise, ambos com 2 cada $(1,3 \%)$ e apenas um $(0,6 \%)$ estudo foi desenvolvido em um centro de reabilitação psicossocial.

Quanto aos sujeitos, 84(52,2\%) estudos envolviam a equipe de enfermagem, 40(24,8\%) estudos abrangeram somente os enfermeiros, 24(14,9\%) abordaram além do pessoal de enfermagem, outros profissionais da equipe multidisciplinar de saúde. Foram encontrados $\quad 5(3,1 \%) \quad$ estudos desenvolvidos com socorristas e a mesma quantidade de pesquisas foi realizada com técnicos de enfermagem. Ainda foram encontrados $3(1,9 \%) \quad$ estudos desenvolvidos com técnicos e auxiliares de enfermagem.

\section{DISCUSSÃO}

O primeiro programa de pósgraduação stricto sensu do Brasil na área da Enfermagem foi criado em 1972 com o primeiro mestrado na Escola de Enfermagem Anna Nery, da Universidade Federal do Rio de Janeiro. Exatamente dez anos depois (1982), iniciava-se o primeiro doutorado em Enfermagem com o Programa Interunidades de Doutoramento, por meio da parceria entre duas unidades da Universidade de São Paulo (USP), a Escola de Enfermagem da capital e a Escola de Enfermagem de Ribeirão Preto. ${ }^{7}$

Atualmente, $\quad \mathrm{o}$ registro da Coordenação de Aperfeiçoamento de Pessoal de Nível Superior (CAPES) indicou a existência de 67 programas de pós-graduação na área da Enfermagem, no Brasil, com um total de 98 cursos, sendo 50 de mestrado acadêmico, 15 de mestrado profissional e 33 de doutorado. ${ }^{8}$ Assim, pode-se observar uma maior concentração de cursos de mestrado, o que explica o número superior de dissertações apresentadas neste estudo.

Em relação ao ano de publicação dos estudos, acredita-se que a publicação de uma nova portaria em 2009 que dispunha acerca da Rede Nacional de Atenção Integral à Saúde do Trabalhador (RENAST) por meio da estruturação da rede de Centros de Referência em Saúde do Trabalhador (CEREST) tenha despertado o interesse pela temática. ${ }^{9}$ Além disso, em 2011, foi instituída a Política Nacional de Segurança e Saúde no Trabalho (PNSST), o que representa um avanço histórico na área, marcada pela luta por direitos da classe trabalhadora, tendo 
como uma de suas estratégias a estruturação da RENAST. ${ }^{10}$

Em 2014 a importação dos dados para a elaboração do catálogo não ocorreu diretamente do Banco de Teses da CAPES, pelo momento de transição da mudança do sistema, com a implantação da plataforma Sucupira. Dessa forma, as informações dos trabalhos defendidos em 2013 e 2014 ainda não estavam disponíveis no Banco de Teses. ${ }^{11}$ Por esse motivo, houve uma redução na publicação de estudos desenvolvidos nesses anos (Gráfico 1).

A maioria dos grupos de pesquisa em Enfermagem cadastrados no Conselho Nacional de Desenvolvimento Científico e Tecnológico está localizada na Região Sudeste, seguida pela Região Sul, Nordeste, Centro-Oeste e Norte. Esses dados refletem tanto o início do crescimento da Pós-Graduação em Enfermagem, quanto à concentração de maior número de Programas de PósGraduação em Enfermagem nas Regiões Sudeste e Sul do país, respectivamente ${ }^{12}$, justificando o número de dissertações e teses encontradas no presente estudo, o que revela a supremacia da região Sudeste (Tabela 1).

Em contrapartida, chama atenção a pequena produção científica na região Norte do país em que precisa investir no desenvolvimento de pesquisas a fim de compreender as nuances e as particularidades acerca da saúde do trabalhador. Trata-se da necessidade de democratização do saber e da gestão do processo de inovação que promova uma possível descentralização por parte das regiões brasileiras à produção nas universidades, com vistas ao equilíbrio regional na formação para o setor de pesquisa em saúde. ${ }^{13}$

Com relação à instituição de saúde de desenvolvimento dos estudos, observase que $70,6 \%$ destes foram realizados em hospitais, seguido de estudos com profissionais de saúde que trabalham nas unidades básicas de saúde (11,8\%). Tal interesse se deve ao fato de que no hospital os riscos e as vulnerabilidades estão relacionados aos acidentes de trabalho com material biológico, à resistência e ao uso inadequado de equipamentos de proteção individual, à sobrecarga de trabalho. Enquanto que, nas unidades básicas de saúde, estão relacionados, principalmente, à deficiência de recursos para realização do trabalho, à violência física e moral e ao desgaste emocional dado pelo contexto sócio-econômico-cultural no qual o trabalho está inserido. ${ }^{14}$

Quanto aos sujeitos, a intencionalidade objetiva dos pesquisadores está voltada, com maior predominância, às investigações com a equipe de enfermagem, que abrangem os enfermeiros, técnicos e auxiliares de 
enfermagem. $^{15}$ Observa-se assim, o interesse em desenvolver pesquisas que avaliem as condições ocupacionais de saúde de todos os trabalhadores de enfermagem das instituições de saúde.

\section{CONCLUSÃO}

Após analisar os 161 resumos desenvolvidos acerca da saúde do trabalhador na pós-graduação stricto sensu brasileira no período de 2009 a 2014, constatou-se que os aspectos predominantes foram: dissertações $(73,91 \%)$, realizados na Região Sudeste $(62,8 \%)$, em hospitais (70,8\%); cujos sujeitos do estudo foram a equipe de enfermagem (52,2\%). Constatou-se que há estudos contínuos sobre saúde do trabalhador ainda que se evidenciem disparidades regionais no processo de produção desse conhecimento.

Como limitação, destaca-se que algumas produções analisadas não apresentaram dados relevantes para sua identificação, como a instituição de saúde em que o estudo foi realizado. Isto indica a necessidade de mais cuidado ao publicálos, pois esta variável constitui informação significativa para fins de caracterização das produções científicas.

Dessa forma, esse estudo traz contribuições não só para a identificação do conhecimento produzido pela enfermagem sobre saúde do trabalhador, mas também fornece subsídios para os programas de pós-graduação no que se refere à análise das informações emitidas para futuras pesquisas.

Espera-se que no futuro venha a existir maior integração entre pesquisadores e gestores para que a pesquisa seja de fato aplicada, visando a melhor adequação das ações na perspectiva da melhoria das condições de trabalho, de vida e da saúde dos trabalhadores.

\section{REFERÊNCIAS}

1. França ISX, Sousa FS, Souto RQ, Coura AS, Araújo AKF, Pagliuça LMF, et al. Ten years of the northeastern nursing network journal - Rev Rene: a dream come true. Rev Rene. [internet]. 2011 [citado em: 16 out 2015]; 12(1):11-7. Disponível em: http://www.revistarene.ufc.br/revista/index .php/revista/article/view/688

2. Presidência da República (BR). Constituição da República Federativa do Brasil de 1988. Brasília: Presidência da República; 1988.

3. Presidência da República (BR). Lei No 8.080, de 19 de setembro de 1990. Dispõe sobre as condições para a promoção, proteção e recuperação da saúde, a organização e $\mathrm{o}$ funcionamento dos serviços correspondentes e dá outras providências. Brasília: Presidência da República; 1990.

4. Ministério da Saúde (BR). Secretaria de Ciência, Tecnologia e Insumos Estratégicos. Departamento de Ciência e Tecnologia. Agenda nacional de prioridades de pesquisa em saúde. Brasília: Ministério da Saúde; 2008.

5. Bezerra MLS, Neves EB. Perfil da Produção Científica em Saúde do Trabalhador. Saúde Soc. [internet]. 2010 
[citado em: 16 out 2015]; 19(2):384-94.

Disponível em:

http://www.revistas.usp.br/sausoc/article/vi ewFile/29655/31525

6. Ribeiro RP, Martins JT, Marziale MHP, Robazzi MLCC. O adoecer pelo trabalho na enfermagem: uma revisão integrativa.

Rev esc enferm USP [internet]. 2012

[citado em: 20 out 2015]; 46(2):495-504.

Disponível em:

http://www.scielo.br/scielo.php?script=sci

_arttext\&pid=S0080-

62342012000200031\&lng=en.

7. Scochi CGS, Munari DB. A pósgraduação em Enfermagem brasileira faz quarenta anos: avanços, desafios e necessidades de novos empreendimentos. [editorial]. Esc. Anna Nery. [internet]. 2012 [citado em: 02 jan 2016]; 16(2):21518. Disponível em:

http://www.scielo.br/scielo.php?script=sci _arttext\&pid=S1414-

$81452012000200001 \& \operatorname{lng}=$ en.

8. Coordenação de Aperfeiçoamento de Pessoal de Nível Superior [homepage na Internet]. Relação de cursos recomendados e reconhecidos. Disponível em: http://conteudoweb.capes.gov.br/conteudo web/ProjetoRelacaoCursosServlet?acao $=p$ esquisarAreaAvaliacao

9. Ministério da Saúde (BR). Portaria $\mathrm{n}^{\circ}$

2.728, de 11 de Novembro de 2009.

Dispõe sobre a Rede Nacional de Atenção

Integral à Saúde do Trabalhador

(RENAST) e dá outras

providências. Brasília: Ministério da

Saúde; 2009.

10. Presidência da República (BR).

Decreto $n^{\circ} 7.602$, de 07 de novembro de 2011. Dispõe sobre a Política Nacional de Segurança e Saúde no Trabalho - PNSST. Brasília: Presidência da República; 2011.

11. Associação Brasileira de Enfermagem.

Centro de Estudos e Pesquisas em enfermagem. Informações sobre pesquisas e pesquisadores em enfermagem.

Brasília: ABEn, 2014. Disponível em: www.abennacional.org.br

12. Costa ACB, Chaves ECL, Terra FS, Monteiro LA. Perfil dos grupos de pesquisa de Enfermagem do Conselho Nacional de Desenvolvimento Científico e Tecnológico. Rev Rene. [internet]. 2014 [citado em: 15 jan 2016]; 15(3):471-9. Disponível em: http://www.revistarene.ufc.br/revista/index .php/revista/article/view/1672/pdf.

13. Backes VMS, Prado ML, Lino MM, Ferraz F, Canever BP, Gomes DC, Martini JG, et al . Teses e dissertações de enfermeiros sobre educação em enfermagem e saúde: um estudo bibliométrico. Rev bras enferm. [internet]. 2013 [citado em: 15 jan 2016]; 66(2):2516. Disponível em: http://www.scielo.br/scielo.php?script=sci _arttext\&pid=S0034$71672013000200015 \& \operatorname{lng}=$ en.

14. Santos JLG, Vieira M, Assuiti LFC, Gomes D, Meirelles BHS, Santos SMA, et al. Risco e vulnerabilidade nas práticas dos profissionais de saúde. Rev. Gaúcha Enferm. (Online) [internet]. 2012 [citado em: 20 jan 2016]; 33(2):205-12.

Disponível em:

http://www.scielo.br/scielo.php?script=sci _arttext\&pid=S1983$14472012000200028 \& \operatorname{lng}=$ en.

15. Custódio IL, Moreira TMM, Lima FET, Freitas MC, Lima MMR, Silva AL, et al. Saúde do trabalhador: caracterização das dissertações e teses nacionais de enfermagem, 2003-2007; Rev enferm UERJ [internet]. 2010[citado em: 15 jan 2016]; 18(4): 604-9. Disponível em: http://www.facenf.uerj.br/v18n4/v18n4a17 .pdf

RECEBIDO: 04/01/2017

APROVADO: $28 / 06 / 2017$

PUBLICADO: $31 / 07 / 2017$ 